\title{
Caught in the Act: Visualization of SNARE-Mediated Fusion Events in Molecular Detail
}

\author{
Herre Jelger Risselada, Carsten Kutzner, and Helmut Grubmüller ${ }^{*[a]}$
}

\begin{abstract}
Neurotransmitter release at the synapse requires fusion of synaptic vesicles with the presynaptic plasma membrane. SNAREs are the core constituents of the protein machinery responsible for this membrane fusion, but the actual fusion mechanism remains unclear. Here, we have simulated neuronal SNARE-mediated membrane fusion in molecular detail. In our simulations, membrane fusion progresses through an inverted micelle fusion intermediate before reaching the hemifused state. We show that at least one single SNARE complex is required for fusion, as has also been confirmed in a recent in vitro singlemolecule fluoresence study. Further, the transmembrane regions of the SNAREs were found to play a vital role in the initiation of fusion by causing distortions of the lipid packing of
\end{abstract}

the outer membrane leaflets, and the $\mathrm{C}$ termini of the transmembrane regions are associated with the formation of the fusion pores. The inherent mechanical stress in the linker region of the SNARE complex was found to drive both the subsequent formation and expansion of fusion pores. Our simulations also revealed that the presence of homodimerizations between the transmembrane regions leads to the formation of unstable fusion intermediates that are under high curvature stress. We show that multiple SNARE complexes mediate membrane fusion in a cooperative and synchronized process. Finally, we show that after fusion, the zipping of the SNAREs extends into the membrane region, in agreement with the recently resolved X-ray structure of the fully assembled state.

\section{Introduction}

SNAREs form a large protein superfamily that consists of small membrane-anchored proteins that mediate membrane fusion reactions in eukaryotes. ${ }^{[1]}$ Complementary sets of opposing SNARE proteins form a tight coil-coiled complex that pulls the opposing membranes together and eventually results in membrane fusion. ${ }^{[2,3]}$ The underlying molecular mechanism, and in particular how this fusion progresses after formation of this complex, is however unclear. ${ }^{[1,3-5]}$ Certain aspects of the fusion mechanism have been linked to the structural characteristics of the SNARE complex. ${ }^{[6,7]}$ In particular, the transmembrane regions (TMR) of the SNAREs were shown to be essential for both inducing membrane merging and subsequent fusion pore formation. ${ }^{[6,8,9]}$ Deletions of TMR end residues have been shown to arrest fusion pore formation. ${ }^{[8]}$ On the other hand the binding affinity between SNAREs and the flexibility of the linkers that connect the TMR with the coiled-coil complex were also shown to play a key role in the formation and expansion of the fusion pore. ${ }^{[7,10]}$ Thus, fusion pore formation seems related to both the presence of mechanical stress in the SNARE complex and the TMR ends.

Here we addressed two main questions: 1) how do the transmembrane regions facilitate both stalk and fusion pore formation? 2) How is the formation of the fusion pore controlled by mechanical stress in the SNARE complex? To this aim, we performed molecular dynamics (MD) simulations of the neuronal SNARE complex, ${ }^{[11]}$ docking the two opposing membranes to reveal physically and energetically feasible fusion pathways after SNARE assembly. In this simulation study we modeled the X-ray-resolved neuronal SNARE complex ${ }^{[11]}$ (see the Supporting Information). For a direct comparison with experiments we set up our simulations in close resemblance to the established in vitro fusion assay, in which complementary sets of SNARE proteins are reconstituted in liposomes, and fusion is studied by fluorescence resonance energy transfer. ${ }^{[1]}$ In vivo, SNAREs mediate fusion within 2-5 ms after arrival of an action potential. ${ }^{[12]}$ In order to capture SNARE-mediated fusion events within the limited simulation timescale (microseconds), we simulated 1) pre-assembled trans-SNARE complexes to allow simulation of the much faster subsequent fusion step, 2) highly fusogenic lipid vesicles (POPE, 1-palmitoyl 2-oleoyl phosphatidylethanolamine) and 3) elevated temperature conditions ( $350 \mathrm{~K}$ instead of $310 \mathrm{~K}$ ). In our simulations upto two neuronal SNARE complexes were embedded in two $20 \mathrm{~nm}$ unilamellar POPE vesicles (Figure 2) and simulated over $4 \mu$ s relative time (Supporting Information).

In this study we synergistically combined the accuracy of conventional atomic simulation models with the speed and efficiency of coarse-grained models (CG), which provide an efficient description by representing multiple atoms by a single interaction site. We performed atomic simulations both to study the membrane partitioning and to validate the accuracy of the MARTINI CG model that we applied ${ }^{[13,14]}$ to overcome

\footnotetext{
[a] H. J. Risselada, C. Kutzner, Prof. H. Grubmüller Theoretical Molecular Biophysics Group, Max-Planck-Institute for Biophysical Chemistry Am Faßberg 11, 37077 Göttingen (Germany) Fax: (+49) 551-201-2302 E-mail: hrissel@gwdg.de

Supporting information for this article is available on the WWW under http://dx.doi.org/10.1002/cbic.201100020: Simulation setup and details.
} 
the large length and timescales involved with capturing SNARE-mediated fusion events. ${ }^{[15,16]}$

\section{Results and Discussion}

In our simulations the assembled coiled-coil SNARE complex is very rigid and exhibits limited conformational flexibility in agreement with earlier atomic simulations and experiments. ${ }^{[17-20]}$ To determine the membrane insertion of the SNARE complex in the bilayer, which is essential to provide a representative setup for the fusion simulations, we exploited the accuracy provided by atomic models and performed several simulations with the SNARE complex embedded in an 128lipid POPE bilayer patch by starting from different insertion depths. Figure $1 \mathrm{~A}$ shows that the insertion depth of the SNARE complex adapts to similar values within $100 \mathrm{~ns}$ despite a difference in initial positioning. Figure $1 \mathrm{~B}$ also shows that the insertion depths obtained with the CG model lies well within the range of the atomic simulations. Thus the insertion depth of the SNARE complex, which is an important and sensitive in-
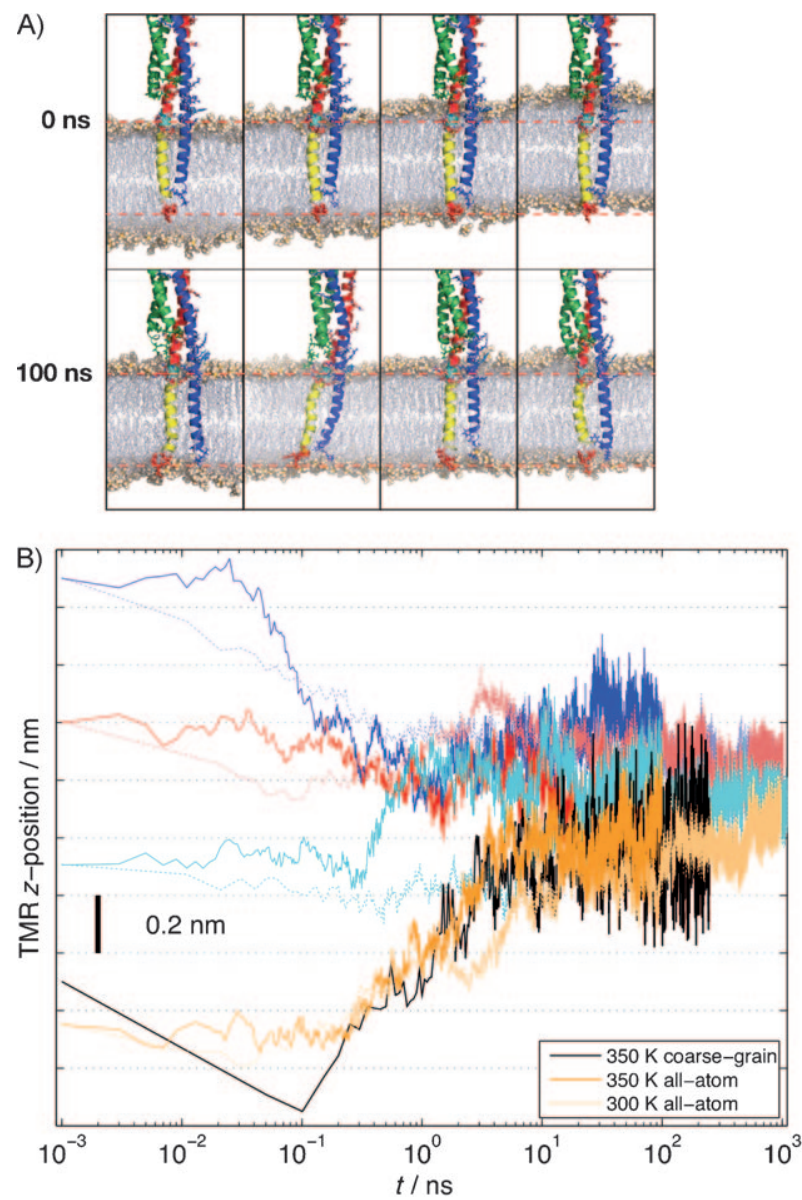

Figure 1. Depth of SNARE complexes in the POPE membrane. A) Adjustment of SNARE complex depth in membrane during the first $100 \mathrm{~ns}$ of the simulation. Pictures have been aligned to the linker region (cyan). B) Distance between the bilayer center and the center of mass of the two trans-membrane regions logarithmically plotted versus time.
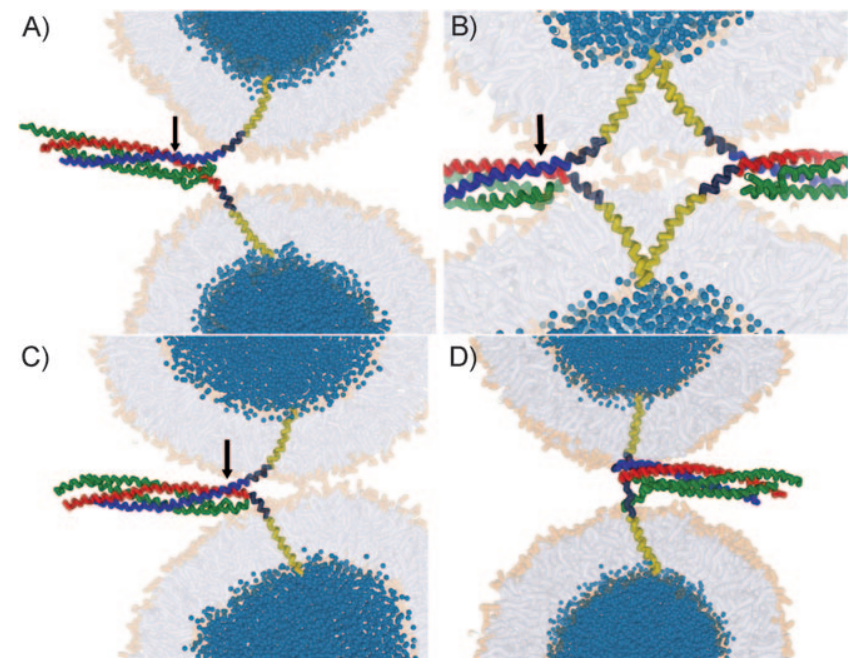

Figure 2. Fusion setups discussed in this work. A, C) Snapshots of the simulation with a single SNARE at different times $t=0.2$ and $3 \mu$ s respectively. These snapshots reveal that a progression of the zipping (black arrows) has pulled the vesicles toward each other. B) Snapshot of the system with two SNARE complexes prior to stalk formation ( $t=0.3 \mu \mathrm{s})$. The zipping (black arrow) has progressed to Lys252 (syntaxin) and Ala56 (synaptobrevin). The TMRs dimerize close to the $C$ termini. D) Snapshot of the system with a single SNARE complex possessing an unstructured linker. Here, the SNARE complex hinders the membranes to come into close proximity. The lipids: the carbon tails are shown in gray, head-groups orange, and (internal) solvent blue. The SNAREs: syntaxin-1A is shown in red, synaptobrevin-2 in blue, and SNAP-25 in green. The SNARE linkers are shown in dark gray and the trans-membrane regions (TMR) in yellow. Internal solvent is shown in blue. For the sake of clarity, exterior solvent is not shown.

dicator of the underlying protein-bilayer interactions, is well reproduced by the CG model. ${ }^{[21]}$

\section{SNARE complex is driven towards the perimeter of the fusion region}

Figure $2 \mathrm{~A}$ and $\mathrm{C}$ reveal that the coarse-grained model of the SNARE complex is mechanical functional and that progression of the zipping pulls the vesicles toward each other. Over the course of microseconds, the single SNARE complex diffuses towards and along the perimeter of the fusion site (vertex ring), maintaining an orientation normal to the perimeter (Figure $2 \mathrm{~A}$, C). Close analysis suggests that mechanical stress stored in the linker region of the SNARE complex plays a key role in this self-organized arrangement. A central positioning of the SNARE complex requires stronger bending of the intrinsically stiff linker regions ${ }^{[22]}$ and would therefore cause a larger mechanical stress. In contrast, a more peripheral positioning of the SNARE complex requires less bending of the linker regions in the SNARE complex and therefore decreases mechanical stress. For the helical syntaxin linker, we estimated a bending stiffness of $10 \pm 0.1 \mathrm{cal} \mathrm{mol}^{-1} \mathrm{deg}^{-2},{ }^{[22]}$ which lies well within the range of the values predicted by atomic simulations (1.7$\left.50 \mathrm{cal} \mathrm{mol}^{-1} \mathrm{deg}^{-2}\right) .{ }^{[22]}$ To prove that mechanical stress stored in the linkers indeed facilitates the observed self-organization, we performed the same simulations by using a SNARE complex with unstructured flexible linkers. Indeed, in these simulations 
the SNARE complex was not driven towards the perimeter of the fusion region (Figure $2 \mathrm{D}$ ). Further, the peripheral positioning of the SNARE complex allows close approach of the two opposing curved membrane patches (Figure $2 \mathrm{~A}, \mathrm{C}$ vs D).

\section{SNARE complexes induce stalk formation and actively opens fusion pores}

Fusion (content mixing) was observed in 4 out of 10 simulations with two SNARE complexes and in two out of ten with a single SNARE complex. The observation that a single SNARE complex is sufficient for membrane fusion agrees with recent experiments. ${ }^{[23,24]}$ In both the single and double SNARE systems fusion progressed through a similar mechanism. The observed fusion mechanism (Figure $3 \mathrm{~A}$ ) shows four main stages:

1) Initial merging of the adjacent leaflets (stalk formation). In our simulations stalk formation is always observed in the direct vicinity of the TMRs of the SNAREs. By what possible mechanism do the TMRs facilitate stalk formation? A detailed look at the lipids close to the TMRs reveals large distortions in the packing of the lipid tails (Figure 4A). Intuitively, these distortions likely enhance the formation of single (Figure 4B) or multiple lipid bridges between the cisleaflets as observed in our simulations, a process that has recently been identified as the kinetic barrier of stalk formation. ${ }^{[25-31]}$ The latter is supported by our SNARE-free control simulation (Supporting Information) in which we artificially brought the vesicles in similar close proximity to each other and stalk formation remained absent (five out of five simulations). In the absence of SNAREs, even when strongly pressing the vesicles against each other (Figure 4C) stalk formation was surprisingly hard to obtain (one out of five simulations). ${ }^{[31-33]}$ In a latter section of this manuscript we
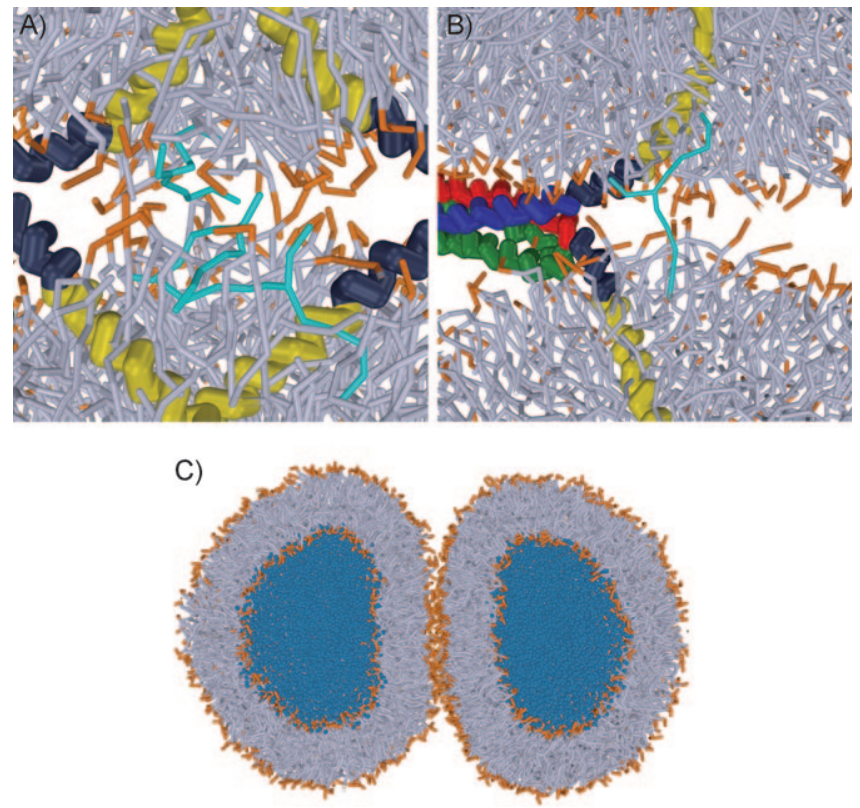

Figure 4. Stalk formation. A) Lipid tail packing in the vicinity of the TMRs. B) A resulting lipid bridge leading to stalk formation. C) SNARE-free control setup with the vesicles strongly pressed against each other.

will look at the stalk-forming propensity of the TMRs in more detail.

2) Formation of an inverted micelle intermediate (IMI). ${ }^{[34]}$ After formation, the stalk expands in a worm-like manner within the cross-section of the fusion plane, eventually closing and forming a ring that encloses an external solvent bubble (Figure $3 \mathrm{~B}$ ). A similar expansion of the stalk is also observed in protein-free fusion simulation studies. ${ }^{[15,26,27]}$ In $3 \mathrm{D}$, the closed ring in fact corresponds to an inverted mi-
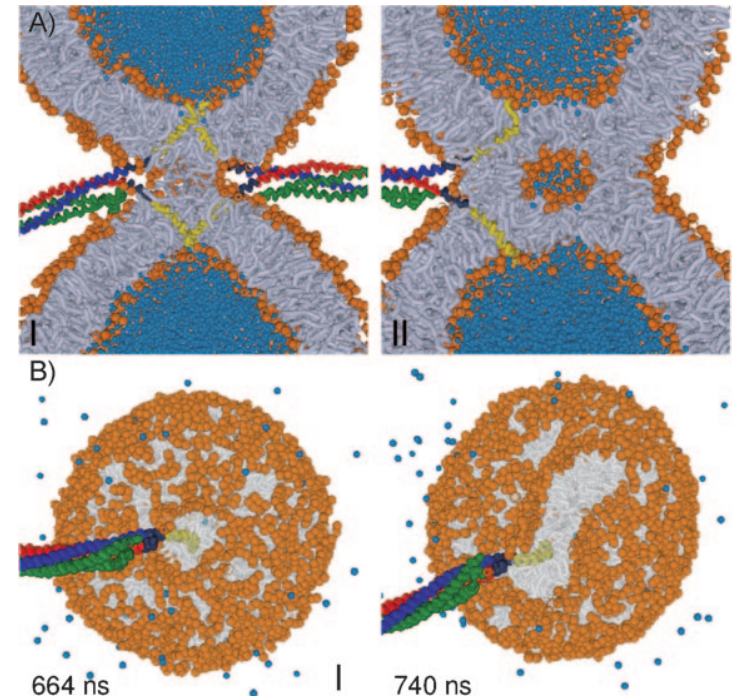
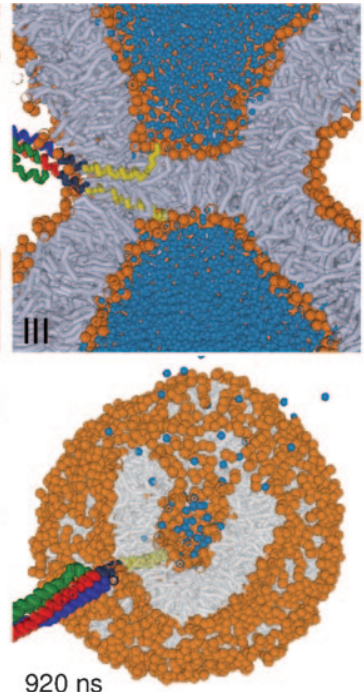

920 ns
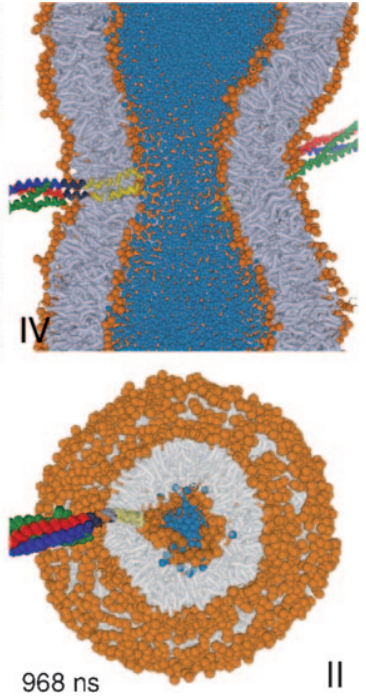

Figure 3. The simulation reveals four stages of fusion. A) I: Stalk, II: inverted micelle intermediate (IMI; single SNARE complex), III: Hemifusion-diaphragm (single SNARE complex), IV: fusion. B) Cross-sections of the fusion plane showing the transition from stage I (stalk) to stage II (inverted micelle) in more detail. The stalk (664 ns), shows a worm-like expansion (740 ns) and eventually, after bending ( $920 \mathrm{~ns})$, forms a closed ring that encapsulates exterior solvent forming an inverted micelle $(968 \mathrm{~ns})$. 
celle (Figure $3 \mathrm{AlI}$ ). The observation that fusion can progress through an IMI before forming a hemifusion diaphragm (Figure 3 A III) has already been predicted by self-consistent field theory. ${ }^{[35]}$ Theory further relates the IMI-pathway, that is, an elongated expansion of the stalk, with the formation of the inverted hexagonal phase. ${ }^{[35]}$ Simulation studies have readily revealed that the lamellar-to-inverted hexagonal phase transition in stacked bilayer systems is indeed facilitated by linear-elongated stalk expansions. ${ }^{[36,37]}$ However, the small circular contact interface between the vesicles enforces bending of the elongated stalk along its perimeter and restricts its topology to that of an inverted micelle. Due to the inherent relation with inverse lipid phases, the formation of IMIs is expected at, for example, a high PE and/or cholesterol content ${ }^{[38,39]}$ or for membranes that are moderately enriched with anionic lipids in the presence of calcium. ${ }^{[40,41]}$ This has readily been observed in simulations for experimentally feasible 1:1 PC/PE mixtures. ${ }^{[42]}$ In support of theory, our simulations suggest a possible experiment to test whether or not the IMI exists: in contrast to the conventional hemifusion pathway, ${ }^{[43,44]}$ encapsulation by the stalk-ring allows vesicular uptake of reasonably large fluorescent molecules ( $3 \mathrm{~nm}$ sized or larger) that reside in the exterior surrounding or the outer membrane surface during fusion and are too large to diffuse through possibly formed leakage pores. Therefore, when fusion progresses through an IMI, we expect an asymmetry between vesicular uptake and release.

3) Formation of the hemifusion diaphragm (HD). When one of the vesicle membranes in the IMI ruptures, the content of the inverted micelle is uptaken (Figure $5 \mathrm{~A}$ ) and an $\mathrm{HD}^{[43,44]}$ remains (Figure $3 \mathrm{AlII}$ ). As a result of curvature stress this membrane is subjected to considerable tension, as evidenced by the reduced membrane thickness in comparison to the vesicle membrane (Figure $3 \mathrm{~A}$ :III). To reduce this tension an HD can alternatively expand, in contrast to the IMI in which expansion is limited by an fixed amount of material enclosed by the micelle.

4) Fusion pore formation. Eventually the HD ruptures, resulting in the formation of a lipidic fusion pore and subsequent content mixing (Figure $3 \mathrm{~A}: \mathrm{IV}$ ). By what possible mechanism can SNAREs facilitate the formation of fusion pores? Figure $5 B$ and $C$ show the early fusion pore. In agreement with current models, ${ }^{[2-4]}$ the $C$ termini of the TMRs reside in or near the appearing fusion pores; this suggests an active role of the SNAREs in the formation of the fusion pores. ${ }^{[45]}$ During hemifusion both synaptobrevin and syntaxin can only release the bending stress stored in the linker region when a fusion pore forms because a large barrier prevents penetration of the charged $C$ termini in the TMRs through the hydrophobic membrane core. Vice-versa, the presence of charged residues in the hydrophobic membrane core, such as the $\mathrm{C}$ termini, has been associated with spontaneous hydrophylic pore formation in atomic simulations. ${ }^{[46]}$ Figure $5 \mathrm{C}$ relates such penetration of the C-terminal to the successive onset of pore formation. Thus, we hypothesize that SNARE complexes open fusion pores, that

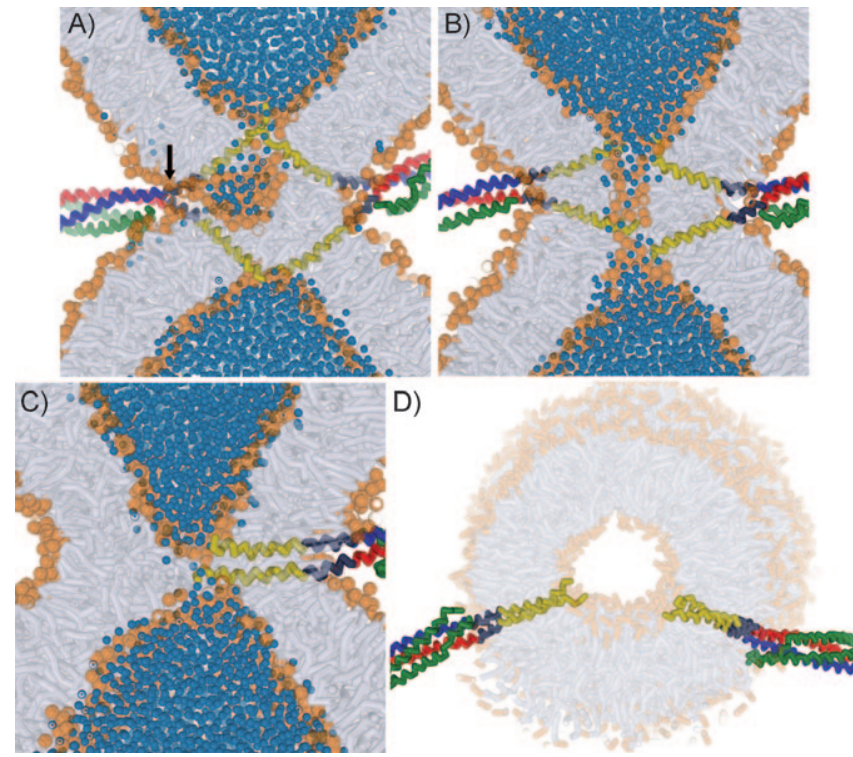

Figure 5. Fusion pore formation. A) Rupture of the first vesicle membrane in the inverted micelle intermediate. The black arrow depicts a leakage pore. B) Formation of the actual fusion pore. C) Penetration of the $C$ terminus into the hydrophobic membrane core triggers pore formation (single SNARE system). D) Cross section of the fused vesicle. Here, the SNARE zipping extends all the way into the membrane region.

this process is driven by the mechanical stress in the SNARE complex that results from SNARE zipping, and that the $C$ termini play an essential role in the underlying mechanism. Should the $\mathrm{C}$ termini indeed be key regulators of the fusion process, our proposed mechanism further predicts that, based on the observations in atomic simulations, ${ }^{[46]}$ any change in their electrostatics is likely to affect the barrier for membrane penetration and thus the inherent pore-forming propensity. Such change could be, for example, chemical modification of the $\mathrm{C}$ terminus, or the addition of net charged lipids. We also note that the presence of C-terminal-bound PHluorins ${ }^{[4]]}$ that were used in the experimental SNARE fusion setups to measure exocytosis might interfere with such a mechanism of pore formation.

\section{Intrinsic properties of trans-membrane regions facilitate stalk formation}

In the previous paragraph we stated that the TMRs facilitate stalk formation by inducing local stress in the bilayer. If such local stress purely arises from mechanical stress stored in the bended SNARE linkers, we would not expect stalk formation if transmission of this bending stress via the TMRs to the membrane is inhibited. Therefore, two simulations were restarted several nanoseconds before the onset of stalk formation, however, now with the structures of both syntaxin and synaptobrevin restrained/frozen in their bent "banana" shape, thus inhibiting the transmission of mechanical stress to the membrane (Supporting Information). Stalks, however, were also formed in both of these simulations (Table S1). Thus, once the SNARE 
complexes have overcome the barrier of bringing the bilayers in close proximity, the subsequent stalk formation is mainly the result of an intrinsic propensity of the TMRs to induce distortion of lipid packing and does not require the presence of mechanical stress in the SNARE complex. The latter agrees with fluorescence studies that showed that isolated SNARE TMR analogues can also trigger membrane fusion. ${ }^{[48]}$ In fact, even if transmission of mechanical stress to the membrane occurs, as already demonstrated, this stress is mainly transduced to the distal trans-leaflets in which the hydrophilic TMR ends reside that open the fusion pore. To illustrate the intrinsic stalk-forming propensity of the TMRs, we performed simulations of the isolated TMRs of both syntaxin and synaptobrevin embedded in a small planar POPC bilayer. Figure 6 shows that

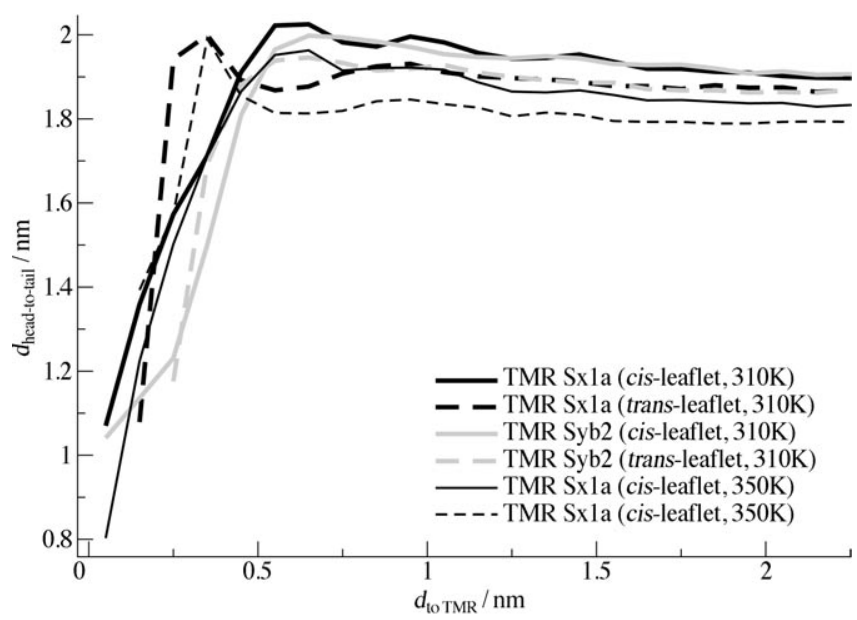

Figure 6. Back-folding of the lipid tails toward the head-group planes as a function of the distance $(d)$ from the TMRs (averaged over $0.5 \mu \mathrm{s}$ ).

both SNARE TMRs (residues 95-116 and 266-288) facilitate strong back-folding of the tail-ends toward the head-group plane within a short range $(<0.7 \mathrm{~nm})$ and weakly disturb the bilayer ordering over a longer range $(3 \mathrm{~nm})$. With respect to the previously observed mechanism of stalk formation (Figure $4 \mathrm{~B}$ ), it is intuitive to associate such a strong local increase in back-folding with an increased propensity of lipid bridge formation between the proximal cis-leaflets. Moreover, the backfolding appeared stronger and longer ranged in the cisleaflets than in the trans-leaflets, and this difference increased with increasing temperatures. The latter suggests that the TMRs possess an inherent directionality, with the N-terminal side being more fusogenic than the $\mathrm{C}$-terminal side. This prediction could be experimentally validated if one could control the direction of bilayer insertion of isolated TMRs in the fusion setup.

\section{Homodimerizations promote the hemifusion to fusion transition}

In our simulations, two SNARE complexes form homodimers between the trans-membrane regions (Figures $2 \mathrm{~B}$ and $8 \mathrm{II}$ ). Similar homodimerizations, close to the $C$ termini of the TMRs, have been observed in the X-ray structure of multiple aggregated neuronal SNARE complexes, ${ }^{[49]}$ further, homodimerizations between TMRs have been shown to promote fusion. ${ }^{[50]}$ What is the underlying mechanism? We focus on the fusion steps that succeed stalk formation, and in particular on the transition from the IMI to the hemifusion intermediate. In simulations in which the stalk ring and its corresponding inverted micelle were small, the rupture of the IMI occurred faster (Table S1). In fact, fusion pore formation is not observed within the $4 \mu$ s simulation when the stalk ring exceeds a diameter of about $8 \mathrm{~nm}$ (Table S1). Remarkably, the size of this stalk ring seems to be strongly linked to the self-organization of the two SNARE complexes (Table S1). In particular, a clustered anti-parallel orientation of the SNAREs in which the SNARE complexes are located opposite to each other, restricts the expansion of the stalk and thus enforces to remain localized (Figure 7). In
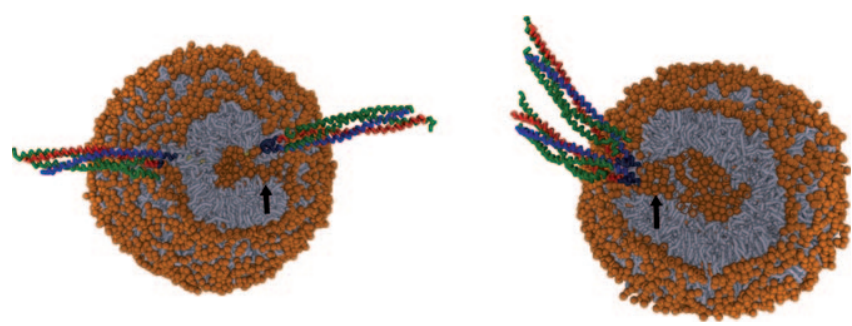

Figure 7. Effect of TMR homodimerizations on stalk expansion. Cross section of the inverted micelle intermediate (stalk-ring) formed with anti-parallel (left) or parallel-aligned (right) SNARE complexes. The black arrows depict a hydrophylic pore that resides in the stalk-ring. Encapsulated solvent is not shown.

contrast, a clustered parallel orientation in which the SNARE complexes are oriented next to each other does not severely hinder the stalk expansion, and thus larger IMls can form (Figure 7). We therefore speculate that homodimerization of the TMRs can decrease the stability of fusion intermediates by localizing the expansion of the stalk forcing the formation of highly stressed fusion intermediates.

\section{SNARE complexes release their energy throughout the whole fusion process up to the expansion of the fusion pore}

Figure 8 reveals that two SNARE complexes mediate vesicle fusion in a correlated/synchronized fashion. This scenario enables multiple SNARE complexes to release energy simultaneously and thus to cooperate in mediating membrane fusion. ${ }^{[24]}$ Remarkably, as an unbiased outcome of the simulation the simulated SNARE complex spontaneously adopts the X-ray structure that has been proposed to represent a post-fusion 


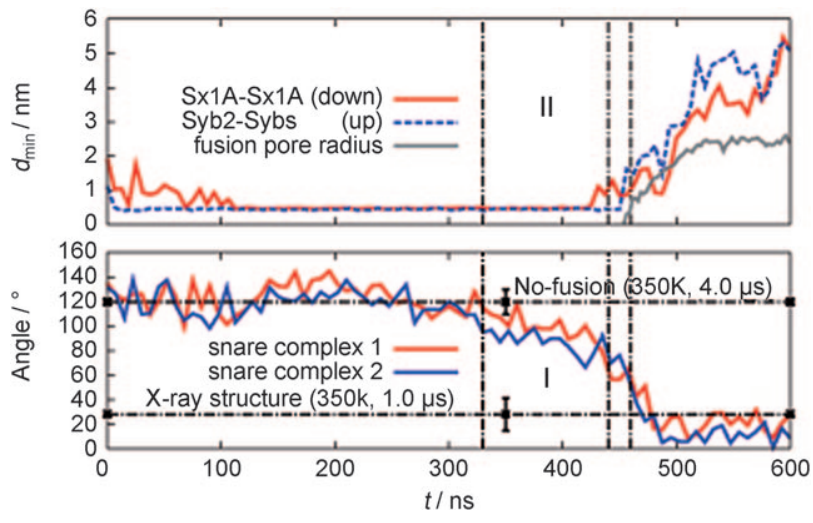

Figure 8. Time evolution of conformational changes in the SNARE complexes during fusion. I) The tilt angle between the TMRs of syntaxin ( $\mathrm{X} \times 1 \mathrm{~A}$, residue 266-288) and synaptobrevin (Syb2, residue 95-116), which represents the magnitude of mechanical stress in the SNARE complex. The horizontal lines represent the average and standard deviation of the tilt angle obtained from the simulation of the SNARE (X-ray structure) embedded in a POPE membrane patch (lower) and two unfused vesicles (upper). II) The minimum contact distance $(d)$ between equal TMRs in different SNARE complexes. A plateau in the minimum contact distance at $\approx 0.5 \mathrm{~nm}$ corresponds to homodimerization between the TMRs. Both TMR species are dimerized between $110-420 \mathrm{~ns}$. Loss of dimerization is related to the onset of membrane rupture. The gray line represents the fusion pore radius. The horizontal lines represent the times of resp. the formation of the stalk, rupture of the IMI and fusion pore.

stage. ${ }^{[11]}$ Similar to this structure, about 40 ns after fusion, both TMRs closely associate, and the SNARE zipping extends all the way into the membrane region (Figure $5 \mathrm{D}$ ). The latter suggests additional progression of SNARE assembly even after fusion. This finding illustrates that the SNARE complex releases its energy gradually and throughout the whole fusion process up to the expansion of the fusion pore. Figure 8 indeed relates the remainder relaxation of the SNARE complexes with the expansion rate of the fusion pore. The latter observation provides further support for the idea that stress released from the SNARE complex participates in driving the expansion of the fusion pore. In such energy transmission, the TMRs progressively dictate decreasing membrane curvature in the pore neck while the SNAREs are relaxing, thus promoting pore expansion. However, because the SNARE complex irreversibly relaxes after fusion pore formation, the observed mousetrap mechanism suggests that a SNARE complex has only "a single shot" in opening the fusion pore.

\section{Acknowledgements}

We thank Reinhard Jahn, Axel Brunger and Geert van den Bogaart for useful discussions and carefully reading the manuscript. We are grateful to the Deutsche Forschungsgemeinschaft, grant SFB 803, for financial support.

Keywords: membranes - molecular modeling simulations neurotransmitters $\cdot$ SNARE $\cdot$ synapses
[1] T. Weber, B. V. Zemelman, J. A. McNew, B. Westermann, M. Gmachl, F. Parlati, T. H. Söllner, J. E. Rothman, Cell 1998, 92, 759-772.

[2] R. B. Sutton, D. Fasshauer, R. Jahn, A. T. Brunger, Nature 1998, 395, 347 353.

[3] R. Jahn, R. H. Scheller, Nat. Rev. Mol. Cell Biol. 2006, 7, 631-643.

[4] R. Jahn, H. Grubmüller, Curr. Opin. Cell Biol. 2002, 14, 488-495.

[5] M. Vrljic, P. Strop, J. A. Ernst, R. B. Sutton, S. Chu, A. T. Brunger, Nat. Struct. Mol. Biol. 2010, 17, 325-331.

[6] D. Langosch, M. Hofmann, C. Ungermann, Cell. Mol. Life Sci. 2007, 64, 850-864.

[7] J. Kesavan, M. Borisovska, D. Bruns, Cell 2007, 131, 351-363.

[8] Y. Xu, F. Zhang, Z. Su, J. A. McNew, Y.-K. Shin, Nat. Struct. Mol. Biol. 2005, $12,417-422$.

[9] J. A. McNew, T Weber, F. Parlati, R. J. Johnston, T. J. Melia, T. H. Söllner, J. E. Rothman, J. Cell Biol. 2000, 150, 105-117.

[10] Q. Fang, K. Berberian, L. W. Gong, I. Hafez, J. B. Sørensen, M. Lindau, Proc. Natl. Acad. Sci. USA 2008, 105, 15388-15392.

[11] A Stein, G. Weber, M. C. Wahl, R. Jahn, Nature 2009, 460, 525-528.

[12] R. Llinas, I. Z. Steinber, K. Walton, Biophys. J. 1981, 33, 323-351.

[13] S. J. Marrink, H. J. Risselada, S. Yefimov, D. P. Tieleman, A. H. de Vries, J. Phys. Chem. B 2007, 111, 7812-7824.

[14] L. Monticelli, S. K. Kandasamy, X. Periole, R. G. Larson, D. P. Tieleman, S. J. Marrink, J. Chem. Theory Comput. 2008, 4, 819-834.

[15] S. J. Marrink, A. E. Mark, J. Am. Chem. Soc. 2003, 125, 11144-11145.

[16] P. M. Kasson, N. W. Kelley, N. Singhal, M. Vrljic, A. T. Brunger, V. S. Pande, Proc. Natl. Acad. Sci. USA 2006, 103, 11916-11921.

[17] T. Hayashi, H. McMahon, S. Yamasaki, T. Binz, Y. Hata, T. C. Sudhof, H. Niemann, J. Biol. Chem. 1994, 13, 5051-5061.

[18] D. Fasshauer, H. Otto, W. Eliason, R. Jahn, A. Brünger, J. Biol. Chem 1997, 272, 28036-28041.

[19] M. P. Durrieu, R. Lavery, M. Baaden, Biophys. J. 2008, 94, 3436-3446.

[20] L. V. Bock, B. Hutchings, H. Grubmüller, D. J. Woodbury, Biophys. J. 2010, 99, $1221-1230$.

[21] M. P. Durrieu, P. J. Bond, M. S. P. Sansom, R. Lavery, M. Baaden, ChemPhysChem 2009, 10, 1548-1552.

[22] V. Knecht, H. Grubmüller, Biophys. J. 2003, 84, 1527-1547.

[23] G. van den Bogaart, M. G. Holt, G. Bunt, D. Riedel, F. S. Wouters, R. Jahn, Nat. Struct. Mol. Biol. 2010, 17, 358-364.

[24] R. Mohrmann, H. de Wit, M. Verhage, E. Neher, J. B. Sørensen, Science 2010, 330, 502-505.

[25] Y. G. Smirnova, S. J. Marrink, R. Lipowsky, V. Knecht, J. Am. Chem. Soc. 2010, 132, 6710-6718.

[26] A. F. Smeijers, A. J. Markvoort, K. Pieterse, P. A. Hilbers, J. Phys. Chem. B 2006, 110, 13212-13219.

[27] M. J. Stevens, J. H. Hoh, T. B. Woolf, Phys. Rev. Lett. 2003, 91, 188102.

[28] A. Efrat, L. V. Chernomordik, M. M. Kozlov, Biophys. J. 2007, 92, L61L63.

[29] A. Grafmüller, J. Shillcock, R. Lipowsky, Biophys. J. 2009, 96, 2658-2675.

[30] P. M. Kasson, E. Lindahl, V.S. Pande, PLoS Comput. Biol. 2007, 6, e1000829.

[31] D. Mirjanian, A. N. Dickey, J. H. How, T. B. Woolf, M. J. Stevens, J. Phys. Chem. B 2010, 114, 11061-11068.

[32] J. C. Shillcock, R. Lipowsky, Nat. Mater. 2005, 4, 225-228.

[33] S. Baoukina, D. P. Tieleman, Biophys. J. 2010, 99, 2134-2142.

[34] D. P. Siegel, Biophys. J. 1993, 65, 2124-2140.

[35] K. Katsov, M. Müller, M. Schick, Biophys. J. 2006, 90, 915-926.

[36] S. J. Marrink, A. E. Mark, Biophys. J. 2004, 87, 3894-3900.

[37] V. Knecht, A. E. Mark, S. J. Marrink, J. Am. Chem. Soc. 2006, 128, $2030-$ 2034.

[38] A. Aeffner, T. Reusch, B. Weinhausen, T. Salditt, Eur. Phys. J. E 2009, 30, $205-214$.

[39] B. G. Tenchov, R. C. MacDonald, D. P. Siegel, Biophys. J. 2006, 91, 2508 2516.

[40] T. S. Awad, Y. Okamoto, S. M. D. Masum, M. Yamazaki, Langmuir 2005 21, $11556-11561$

[41] A. Yaghmur, P. Laggner, B. Sartori, M. Rappolt, PLOS 2008, 3, e2072.

[42] Marrink. S. J. , A. H. de Vries, D. P. Tieleman, Biochim. Biophys. Acta Biomembr. 2009, 1788, 149-168.

[43] M. M. Kozlov, V. S. Markin, Biofizika 1983, 28, 255-261.

[44] L. V. Chernomordik, M. M. Kozlov, Annu. Rev. Biochem. 2003, 72, 175 207.

[45] S. Wu, H. Guo, J. Phys. Chem. B 2009, 113, 589-591. 
[46] D. P. Tieleman, S. J. Marrink, J. Am. Chem. Soc. 2006, 128, 12462-12467.

[47] G. Miesenböck, D. A. De Angelis, J. E. Rothman, Nature 1998, 394, 192 195.

[48] D. Langosch, J. M. Crane, B. Brosig, A. Hellwig, L. K. Tamm, J. Reed, J. Mol. Biol. 2001, 311, 709-721.

[49] A Stein, G. Weber, M. C. Wahl, R. Jahn, Nature 2009, Suppl., 1-6.
[50] M. W. Hofmann, K. Peplowska, J. Rohde, B. Poschner, C. Ungermann, D. Langosch, J. Mol. Biol. 2006, 364, 1048-1060.

Received: January 11, 2011

Published online on March 23, 2011 